\title{
Successful Management of an MRSA Outbreak in a Neonatal Intensive Care Unit
}

Norbert Heinrich, MD;Andreas Mueller, MD; PhD;Peter Bartmann, MD, Professor;Arne Simon, MD, PhD;Gabriele Bierbaum, Professor;Steffen Engelhart, MD; PhD

Klinikum of the University of Munich, Munich, Germany Corresponding Author: Norbert Heinrich, MD

Abstract: We report an MRSA outbreak in our 25 bed tertiary neonatal intensive care unit (NICU), which was successfully contained.

Patients and Methods: retrospective review of patient files, microbiology records and meeting protocols.

Results: During the seven months of outbreak, 27 patients and 7 health care workers (HCWs) had positive cultures for MRSA. The outbreak was caused by the epidemic Rhine-Hessen strain, cultured isolates were monoclonal. After a sharp increase of the number of new MRSA-cases the installation of an outbreak management team (OMT) and implementation of comprehensive measures (extensive screening and decolonization strategy including orally applied vancomycin, isolation wards, intensive disinfection regimen) successfully terminated the outbreak within one month. Ten (53\%) of 19 patients with completed follow up and all of the HCWs were decolonized successfully. Gastrointestinal colonization was present in 15 of $27(56 \%)$ neonates, and was associated with poor decolonization success ( $30 \%$ vs. $78 \%$ in absence of gastrointestinal colonization);

Conclusion: A comprehensive outbreak management can terminate an outbreak in a NICU setting within a short time. Thorough screening of nares, throat and especially stool is necessary for correct cohorting. Gastrointestinal decolonization in neonates seems difficult.

Keywords: Methicillin-resistant Staphylococcus aureus; MRSA; outbreak management; neonates; NICU; gastrointestinal colonization

\section{INTRODUCTION}

The immaturity of the immune system, use of broad spectrum antibiotics, surgical procedures, invasive devices and mechanical ventilation expose neonatal intensive care patients to an increased risk 
of colonization and infection with multidrug-resistant pathogens [1-2]. Among these, outbreaks caused by Methicillin-resistant Staphylococcus aureus (MRSA) are increasingly reported, causing significant morbidity and mortality in neonatal intensive care units (NICUs) [3] In 2006, Gerber et al. published a consensus paper from the Chicago-Area Neonatal MRSA Working Group on containment and prevention measures, stating that hospitals took substantially different approaches for MRSA control [4].Prospectively randomized trials comparing different methods of screening and decolonization treatment are lacking in this high risk neonatal population [5-6].

This article discusses our experiences and conclusions related to the successful containment of an MRSA outbreak involving 27 neonates and 7 health care workers in a German level III NICU. 


\section{MATERIALS AND METHODS}

\section{Setting and study population}

The Department of Neonatology of the University of Bonn is a tertiary neonatal care unit, providing 25 beds in a level III NICU and a neonatal intermediate care unit (NIMC), which are situated at a distance of about $8 \mathrm{~km}$. Approximately 400 infants are admitted per year. All neonates admitted to the NICU during the outbreak period were included into this retrospective analysis. Basic clinical patient data of MRSA-colonized infants were extracted from patients' files. Microbiological results were obtained from the database of the hospital laboratory (Institute of Medical Microbiology, Immunology and Parasitology, University of Bonn). All patients with at least one culture positive for MRSA were included. Decontamination cycles were counted, as well as treatment cycles with oral vancomycin and intravenous linezolid. All patients with at least one MRSA-positive stool sample were classified as gastrointestinal carriers ('Gl-positive'), whereas children with at least two negative stool samples were classified as 'GI negative'.

\section{Cohorting, Isolation, and specific Disinfection Measures}

The outbreak was divided into an "endemic" phase from February to October 2005, with 15 patients diagnosed as MRSA positive during that 
period, and an "epidemic" phase from November 2005 to January 2006, with 12 patients newly diagnosed in November only, and no patients newly identified thereafter.

During the endemic phase, preventive measures included isolation or barrier nursing of patients, a hygiene training and voluntary screening of health care workers, routine disinfection measures (usual hand disinfection, wiping technique for surfaces), and environmental investigations by contact plate samples from diverse hand-contact and skin-contact surfaces in the patient's immediate environment.

The sharp increase in case number in November prompted more intensive measures. An outbreak management team (OMT) was installed in order to implement a detailed concept for screening, hygiene, decontamination and cohorting. The NIMC ward was exclusively used to cohort all patients with known MRSA colonization ("isolation ward"). All non-colonized children were transferred to the NICU, in which all surfaces had been thoroughly disinfected using the spray-fogging technique with terminal wiping of surfaces. After release of MRSA patients from the NIMC ward, rooms were disinfected in the same fashion. Patients newly admitted to the NICU were isolated in a single room, until their MRSA carrier state was determined.

In addition to hand disinfection, staff and family members were instructed to wear gloves, gowns, and a surgical face mask during any contact to 
an MRSA-colonized patient or to any potential fomites in the patient's vicinity.

\section{MRSA Screening of Neonates}

Before the outbreak, routine MRSA screening was not practised. Once installed, the OMT implemented screening of nasal, pharyngeal, and rectal swabs and stool specimens sampled $24 \mathrm{~h}$ after admission, and then once weekly.

Three days after the completion of each cycle of decolonization treatment, swabs were taken from the nares, throat, axilla, anus, groins, and stool three times on consecutive days to assess decolonization success.

\section{MRSA screening of Health Care Workers}

Health care workers were screened by nasal and pharyngeal swabs, mandatory screening was introduced during the epidemic phase.

\section{Microbiology}

Microbiological analysis of swabs was performed using MRSASelect agar (Bio-Rad Laboratories, Marnes-la-Coquette, France), a chromogenic medium, which delivers rapid differentiation based on the activity of mecA gene product. 


\section{Pulsed field gel electrophoresis (PFGE)}

Chromosomal DNA for the smal (Roche, Germany) restriction digests was purified as described previously (Goering and Winters, 1992). Pulsed-field gel electrophoresis (PFGE) was performed on the Chef DRIII system (Bio-Rad, Germany) using Pulsed Field Certified Agarose (1\%) (Bio-Rad), $6 \mathrm{~V} / \mathrm{cm}$, a field angle of $120^{\circ}$, a switch time of $5-15 \mathrm{~s}$ for 7 $\mathrm{h}$ and $15-60 \mathrm{~s}$ for a further $19 \mathrm{~h}$. Staphylococcus aureus NCTC 8325 served as a standard.

\section{Phage typing}

Phage typing was performed with the international phage typing set (IPS) (group I: 29, 52, 52A, 79, 80; group II: 3A, 3C, 55, 71; group III: 6, 42E, $47,53,54,75,77,83 \mathrm{~A}, 84,85$; group V: 94, 96; group M: 81, 95) for $S$. aureus at $100 \mathrm{x}$ routine test dilution. Additionally, the MRSA phage typing set (F30, F33, F38, MR8, MR12, MR25, M3, M5, 622, 56B) and additional regional phages (D11, 19 92, 187 and 192) were employed. Phage types were determined by the strong reactions according to the international rules. Two type strains of the Rhine-Hessen strain ( $S$. aureus 3391/02 and 2981/04) were received from the German National Reference Center for staphylococci, Wernigerode. 


\section{Decolonization Regimen for Patients and HCWs}

Total body washing of colonized neonates with diluted polyhexanide (Sanalind $^{\circledR}$, Fresenius KABI, Bad Homburg, Germany) was done once daily. Mupirocin cream (Turixin ${ }^{\circledR}$, GlaxoSmithKline, Munich, Germany) was applied to the nares three times per day for five days. In selected patients with persistent gastrointestinal colonization, vancomycin was given orally (30 mg/kg/d in three single doses) for 10 days, together with Lactobazillus GG capsules.

Decolonization of HCWs included nasal mupirocin, throat and skin application of polihexanide and/or octenidine, daily change and washing $\left(>60^{\circ} \mathrm{C}\right)$ of clothes and bedding, instruction in hand disinfection techniques, and daily disinfection of hand-contact surfaces (working and home setting).

\section{Statistical analysis}

Data were analysed using Epi Info (version 6; CDC, Atlanta, GA) and rates were compared using the chi-square test or Fisher's exact test as appropriate. 


\section{RESULTS}

The outbreak lasted for 11 months (Feb 2005-Jan 2006) and involved 27 patients. During the following year, no MRSA was detected in any patient in the NICU. Of 358 admissions during the outbreak period, 27 (7.5\%) patients had at least one culture positive for MRSA. None of these patients were transferred in from an external hospital. The 27 MRSApositive patients had a median birth weight of $1,550 \mathrm{~g}$ (interquartile range, IQR, 1,073-1,765g); the median gestational age at birth was 31 weeks (IQR, 29-34 weeks). The median duration of hospitalization was 51 days (IQR, 25,5-83 days). In none of these patients, the length of stay in hospital was prolonged as a result of MRSA colonization or infection.

The index patient was born at $34+0$ weeks of gestation. She suffered from congenital generalized lymphangiectasia, and required long-term mechanical ventilation. MRSA was first detected in ascites and pleural effusion during a systemic infection after retransfer from surgery. Intravenous treatment with Linezolid resulted in negative culture results 4 days later. Surveillance cultures remained negative for three weeks; afterwards MRSA was again cultivated from her nares. In the following course, swabs and ascites cultures remained negative until the patient died from her underlying disease at day 309 of her hospitalization.

In the other colonized patients, no symptoms attributable to MRSA were detected, apart from mild conjunctivitis in one patient. 


\section{Neonatal Screening Results and Sampling Site}

MRSA was most frequently detected in nasal swabs followed by specimens from throat and stool.

Swabs from skin (axilla, groins, anus) did not contribute to detection of persistent colonization. Screening of stool, nares and throat would have detected all 25 episodes of persistent colonization. Screening of nares and throat only would have missed five episodes (19\%) of persistent colonization.

To assess the possibility of taking less than three swabs or samples per site for detection of persistent colonisation, the number of swabs positive for MRSA out of three was counted. From 21 episodes with three swabs taken on successive days per sampling site, 1 of 3 swabs was positive in 11 episodes, 2 of three in 4 episodes, and 3 of 3 in 6 episodes.

\section{Screening Results in Health Care Workers and Environment}

During the endemic phase, no MRSA was isolated from $60 \mathrm{HCW}$ screenings. From 118 environmental samples taken, MRSA was isolated twice from a stethoscope. During the epidemic phase, no MRSA was isolated from 42 environmental samples; $142 \mathrm{HCW}$ sere screened, nine persons $(6.3 \%$, five nurses, three nursing students and one ward assistant responsible for environmental cleaning and disinfection 
procedures in the NICU) were identified as nasal and/or throat MRSA carriers. However, only seven isolates (4.9\%) were related to the outbreak isolate. All staff members were successfully decolonized. Two nurses had to undergo a second cycle of mupirocin, one nurse suffering from atopic dermatitis was successfully decolonized after three cycles. Due to underlying bronchial asthma, disinfection-related coughing and throat colonization with MRSA, the ward assistant was assigned to a workplace outside of neonatology department.

\section{Phage Typing, PFGE and in vitro Antibiotic Sensitivity}

All of the 27 neonatal and seven of the nine HCW-related outbreak isolates showed strong reactions in phage group III $(47,54,75,77,83)$ and group $M(81)$ of the IPS, with the additional regional phages (D11, 16, 192) and the MRSA phage set (MR8, MR12 and M3). This pattern characterized the isolates as epidemic Rhine-Hessen clone of hospitalacquired MRSA. All isolates tested showed a pattern characteristic to the epidemic Rhine-Hessen clone MRSA.

All strains were resistant to B-lactams, erythromycin, clindamycin and ciprofloxacin in vitro, in accordance with the usual phenotype of hospitalacquired MRSA.

\section{Decolonization Success in Neonates}


Of the 27 neonates with positive MRSA cultures, 8 patients were discharged or transferred to other units as 'MRSA positive' before completion of decolonization. Of the 19 remaining patients with available results after decolonization, 9 (47\%) showed persistent colonization and $10(53 \%)$ were decolonized successfully.

One standard cycle of mupirocin and polyhexanide bathing was sufficient to decolonize seven children, one of whom received additional oral vancomycin. Two patients were decolonized after two cycles. More than two cycles were applied to three children, but without success.

Gastrointestinal colonization was detected in 15 of 27 (56\%) neonates. Sufficient follow-up data after decolonisation was available for 10,3 of whom were decolonised successfully (i.e., $30 \%$ vs. $78 \%$ in absence of gastrointestinal colonization; $R R=0,39$ [95\% Confidence interval: 0.14 1.06]).

Vancomycin was applied orally to seven patients with GI-positive cultures. In only one of these, a successful decolonization was confirmed by cultures.

\section{DISCUSSION}

The hospital-acquired MRSA outbreak in our NICU was caused by a single clone, confirmed by phage and PFGE-typing. We assume a combination of indirect patient-to-patient transmission and spread 
through the colonization of health care workers as previously described [7], which may have been supported by insufficient adherence to standard hygiene procedures and the ability of MRSA to persist for long periods on contaminated surfaces [8-9]. Fortunately, no patient other than the index case suffered a serious infection due to MRSA. During an initial endemic phase (February to October) with a mean of $1.7(0-4)$ new MRSA-cases/month, standard preventive measures were taken. The installation of an outbreak management team (OMT), prompted by a sharp increase in incidence, including the decision-makers of the unit helped to implement measures successfully. We assume that our comprehensive approach targeting patients, staff and environment as possible reservoirs for MRSA (cohorting on isolation wards, comprehensive disinfection measures, extensive screening and decontamination strategy) was important for the successful termination of the outbreak.

Since December 2005, one month after installation of the OMT, no single new MRSA-case was registered for more than a year.

In contrast to other authors, who declared nasal swabs as the 'one and only' screening site [5], our data show a higher sensitivity of stool cultures, in particular in the detection of persisting carriage, and suggest screening of nares, thorat and stool. The gastrointestinal tract has been identified as an important reservoir of MRSA in persistent carriers in 
adult patients [10-14], and in a pediatric intensive care unit [15]. Contrary to the data reviewed by Acton et al, rectal swabs were clearly inferior to stool samples in detecting persistent colonization [14].

We assume that gastrointestinal colonization in neonates contributes significantly to the overall risk of environmental contamination, particularly if standard hygiene procedures and routine surface disinfection are not strictly followed [8-9]. The approach to administer vancomycin to GI-positive patients [15] has been disappointing in our patients.

In screening for persistent colonisation, we advise against using less than three swabs per site, since in the majority of episodes only one swab out of three yielded MRSA. Although health care workers (HCW) have been involved in outbreaks [7], the role of routine HCW-screening and the question how to handle a positive result in a HCW is still a matter of debate. However, screening and decolonization of HCWs and exclusion from direct patient care may play an important role. The detection of MRSA colonization in a woman (presenting with bronchial asthma, throat MRSA colonization, and disinfection-associated coughing) responsible for surface cleaning and disinfection efforts in our unit underlines the necessity to include not only nurses and physicians into the screening population. 
In summary, we conclude that a comprehensive outbreak management including the decision-makers can successfully terminate an outbreak in a NICU setting within short time. Thorough screening of nares, throat and stool for MRSA, repeated three times, is necessary for correct cohorting of patients. However, gastrointestinal decolonization in neonates seems difficult. 


\section{ACKNOWLEDGEMENT}

The authors thank the nursing staff of the Department of Neonatology, the company medical officer, and the clinical staff of the Department of Hospital Hygiene for their contribution to control this outbreak.

Financial support: No financial support

Potential conflict of interest: All authors no conflict 


\section{REFERENCES}

1. Chuang YY, Huang YC, Lee CY, Lin TY, Lien R, Chou YH (2004) Methicillin-resistant Staphylococcus aureus bacteraemia in neonatal intensive care units: an analysis of 90 episodes. Acta Paediatr 93 (6):786-790

2. Adams-Chapman I, Stoll BJ (2002) Prevention of nosocomial infections in the neonatal intensive care unit. Curr Opin Pediatr 14 (2):157-164

3. Reboli AC, John JF, Jr., Levkoff AH (1989) Epidemic methicillingentamicin-resistant Staphylococcus aureus in a neonatal intensive care unit. Am J Dis Child 143 (1):34-39

4. Gerber SI, Jones RC, Scott MV, Price JS, Dworkin MS, Filippell MB, Rearick T, Pur SL, McAuley JB, Lavin MA, Welbel SF, Garcia-Houchins S, Bova JL, Weber SG, Arnow PM, Englund JA, Gavin PJ, Fisher AG, Thomson RB, Vescio T, Chou T, Johnson DC, Fry MB, Molloy AH, Bardowski L, Noskin GA (2006) Management of outbreaks of methicillinresistant Staphylococcus aureus infection in the neonatal intensive care unit: a consensus statement. Infect Control Hosp Epidemiol 27 (2):139145. doi:ICHE2005124 [pii]

$10.1086 / 501216$

5. Singh K, Gavin PJ, Vescio T, Thomson Jr RB, Jr., Deddish RB, Fisher A, Noskin GA, Peterson LR (2003) Microbiologic surveillance using nasal cultures alone is sufficient for detection of methicillin-resistant Staphylococcus aureus isolates in neonates. J Clin Microbiol 41 (6):2755-2757

6. Hitomi S, Kubota M, Mori N, Baba S, Yano H, Okuzumi K, Kimura S (2000) Control of a methicillin-resistant Staphylococcus aureus outbreak in a neonatal intensive care unit by unselective use of nasal mupirocin ointment. J Hosp Infect 46 (2):123-129. doi:10.1053/jhin.2000.0786 S0195-6701(00)90786-2 [pii]

7. Bertin ML, Vinski J, Schmitt S, Sabella C, Danziger-Isakov L, McHugh M, Procop GW, Hall G, Gordon SM, Goldfarb J (2006) Outbreak of methicillin-resistant Staphylococcus aureus colonization and infection in a neonatal intensive care unit epidemiologically linked to a healthcare 
worker with chronic otitis. Infect Control Hosp Epidemiol 27 (6):581-585. doi:ICHE2005248 [pii]

$10.1086 / 504933$

8. Hota B (2004) Contamination, disinfection, and cross-colonization: are hospital surfaces reservoirs for nosocomial infection? Clin Infect Dis 39

(8):1182-1189. doi:CID33640 [pii]

$10.1086 / 424667$

9. Dancer SJ (2008) Importance of the environment in meticillin-resistant Staphylococcus aureus acquisition: the case for hospital cleaning. Lancet Infect Dis 8 (2):101-113. doi:S1473-3099(07)70241-4 [pii] 10.1016/S1473-3099(07)70241-4

10. Donskey CJ (2004) The role of the intestinal tract as a reservoir and source for transmission of nosocomial pathogens. Clin Infect Dis 39 (2):219-226. doi:10.1086/422002 CID33097 [pii]

11. Klotz M, Zimmermann S, Opper S, Heeg K, Mutters R (2005) Possible risk for re-colonization with methicillin-resistant Staphylococcus aureus (MRSA) by faecal transmission. Int J Hyg Environ Health 208 (5):401-405

12. Boyce JM, Havill NL, Maria B (2005) Frequency and possible infection control implications of gastrointestinal colonization with methicillin-resistant Staphylococcus aureus. J Clin Microbiol 43 (12):5992-5995. doi:43/12/5992 [pii]

10.1128/JCM.43.12.5992-5995.2005

13. Boyce JM, Havill NL, Otter JA, Adams NM (2007) Widespread environmental contamination associated with patients with diarrhea and methicillin-resistant Staphylococcus aureus colonization of the gastrointestinal tract. Infect Control Hosp Epidemiol 28 (10):1142-1147. doi:ICHE2006422 [pii]

$10.1086 / 520737$

14. Acton DS, Plat-Sinnige MJ, van Wamel W, de Groot N, van Belkum A (2009) Intestinal carriage of Staphylococcus aureus: how does its frequency compare with that of nasal carriage and what is its clinical impact? Eur J Clin Microbiol Infect Dis 28 (2):115-127. doi:10.1007/s10096-008-0602-7

15. Thorburn K, Taylor N, Saladi SM, van Saene HK (2006) Use of surveillance cultures and enteral vancomycin to control methicillin- 
resistant Staphylococcus aureus in a paediatric intensive care unit. Clin Microbiol Infect 12 (1):35-42. doi:CLM1292 [pii] 10.1111/j.1469-0691.2005.01292.x 